\title{
Sphingomyelin synthase 2 overexpression promotes cisplatin-induced apoptosis of HepG2 cells
}

\author{
SI LUO, ZHEN PAN, SHUANG LIU, SHUJING YUAN and NIANLONG YAN \\ Department of Biochemistry and Molecular Biology, School of Basic Medical Science, \\ Nanchang University, Nanchang, Jiangxi 330006, P.R. China
}

Received April 29, 2016; Accepted September 15, 2017

DOI: $10.3892 / \mathrm{ol} .2017 .7309$

\begin{abstract}
Hepatoblastoma (HB) is the most type of common pediatric liver cancer. The primary chemotherapy drug for $\mathrm{HB}$ is cisplatin (DDP). However, patients readily develop intrinsic and acquired resistance, and severe side effects to treatment. Sphingomyelin synthase 2 (SMS2) is a key enzyme involved in the generation of sphingomyelin (SM), which is able to regulate cell proliferation, apoptosis and differentiation. The death receptors (DRs) have important functions in DDP-induced apoptosis. However, whether SMS2 is able to modulate cell apoptosis through the DR signaling pathway remains unknown. To investigate this question, SMS2 was overexpressed in HepG2 cells and treated with $3.5 \mathrm{mg} / 1$ cisplatin in the present study. After $24 \mathrm{~h}$, the expression of SMS2, avian myelocytomatosis viral oncogene homolog (c-Myc), DR4, DR5 and caspase-3 was analyzed. Furthermore, cell viability was quantified, and apoptosis was assessed by western blot and flow cytometry analysis as well as Cell Counting kit-8. The results of the present study revealed that overexpression of SMS2 was able to increase the expression of c-Myc, cleaved caspase-3, DR4 and DR5 compared with the control group $(\mathrm{P}<0.05, \mathrm{n}=3)$, and increase the levels of apoptosis in the SMS2 + DDP group, compared with the control $(\mathrm{P}<0.001, \mathrm{n}=3)$. These results indicate that overexpression of SMS2 is able to improve sensitivity of HepG2 cells to DDP by increasing the expression of c-Myc, DR4 and DR5 in HepG2 cells. This increased sensitivity may decrease intrinsic and acquired resistance of chemotherapy in $\mathrm{HB}$, and reduce the associated severe side effects in pediatric patients.
\end{abstract}

Correspondence to: Professor Nianlong Yan, Department of Biochemistry and Molecular Biology, School of Basic Medical Science, Nanchang University, 461 Bayi Road, Nanchang, Jiangxi 330006, P.R. China

E-mail: yannianlong@163.com

Key words: cisplatin, sphingomyelin synthase 2, death receptor 4, death receptor 5, HepG2, hepatoblastoma

\section{Introduction}

Hepatoblastoma (HB) is a malignant liver tumor observed in pediatric patients, the incidence of which has increased by $2.18 \%$ annually in patients $<20$ years of age (1). A total of $90 \%$ of patients with liver malignancies and $<5$ years of age were diagnosed with HB (1). Previously, the main treatment for HB was surgical resection. However, complete tumor resection may only be achieved in a small proportion of patients (1). However, since cisplatin (DDP) was introduced into the treatment regimen, the survival rate had improved markedly (1). DDP is a platinum-based chemotherapeutic that belongs to a class of alkylating agents widely used in the treatment of a variety of pediatric malignancies (2). However, as occurs with the majority of anticancer drugs, treatment resistance and side effects (including hearing loss) in healthy tissues are two major challenges in the use of $\operatorname{DDP}(2,3)$. The DDP-associated toxicity is dose-dependent, but inhibition of cancer cell proliferation requires a sufficient dose of DDP. Therefore, there exists a contradiction between chemotherapy efficiency and side effects, meaning that there is an urgent requirement to enhance the sensitivity of DDP chemotherapy.

The anticancer mechanism of DDP is associated with its ability to form inter- and intra-strand DNA crosslinks, which perturb DNA replication and transcription, therefore inducing a replication stress and DNA damage response, eventually resulting in cell cycle arrest and apoptosis (4). Apoptosis induced by DDP can be initiated through two main core signaling pathways, the tumor protein 53-dependent transcription of pro-apoptotic B-cell lymphoma-2 family members (p53 upregulated modulator of apoptosis, phorbol-12-myristate-13-acetate-induced protein 1 and Bcl-associated $\mathrm{X}$ ) that trigger cell death via the mitochondrial apoptotic pathway and the death receptors (DRs) (5). DRs are receptors of tumor necrosis factor-related apoptosis-inducing ligands (TRAILs), which have five receptors. However, only two of the receptors, DR4 (TRAIL-R1) and DR5 (TRAIL-R2), are capable of effectively transmitting the apoptotic signal (5). A number of studies reported that DDP enhances the sensitivity of TRAIL to the cancer cells by increasing the expression of DR4 and DR5 $(6,7)$, which triggers the relocalization of DR4 and DR5 to the cell membrane and accelerates the internalization of TRAIL $(2,6,8)$. However, DDP can also induce apoptosis through the DR4 and DR5 signaling pathways, which is 
not dependent on TRAIL and the mitochondrial apoptotic signaling pathway (9).

Sphingomyelin synthase (SMS) is a key enzyme involved in the generation of sphingomyelin (SM) and has two isoforms, SMS1 and SMS2 (10). SMS can participate in inflammation, atherosclerosis, proliferation, apoptosis, differentiation and other functions $(11,12)$. However, the association between SMS2 with the expression of DR4 and DR5, and DDP-induced apoptosis is unclear in HepG2 cells.

Therefore, the present study constructed a SMS2 overexpression cell model to investigate the effects of SMS2 on the expression of DR4 and DR5, and on apoptosis induced by DDP. HepG2 cells were previously misidentified as human hepatocarcinoma (13); however, in the present study, HepG2 cells were treated as $\mathrm{HB}$, and the results were not affected by this misidentification.

\section{Materials and methods}

Cell culture and transfection. HepG2 cells were cultured in Dulbecco's modified Eagle's medium (DMEM; Hyclone; Thermo Fisher Scientific, Inc., Waltham, MA, USA) supplemented with $10 \%$ fetal bovine serum (FBS; Zhejiang Tianhang Biotechnology Co., Ltd., Huzhou, China) and penicillin and streptomycin $(100 \mathrm{U} / \mathrm{ml}$ and $0.1 \mathrm{mg} / \mathrm{ml}$, respectively; Beijing Solarbio Bioscience \& Technology Co., Ltd., Beijing, China), and incubated at $37^{\circ} \mathrm{C}$ in a humidified atmosphere containing $5 \% \mathrm{CO}_{2}$.

Transfection was performed using LipoFiter transfection reagent (Hanbio Biotechnology Co., Ltd., Shanghai, China) according to the manufacturer's protocol. At $12 \mathrm{~h}$ before transfection, the cells $\left(7 \times 10^{5}\right)$ were seeded into wells of a 6 -well plate that contained antibiotic-free DMEM. At the time of transfection, cell confluence was $70-80 \%$. The SMS2 plasmid (4 $\mu \mathrm{g}$; provided by Dr Tingbo Ding, School of Pharmacy, Fudan University, Shanghai, China) or negative plasmid (4 $\mu \mathrm{g}$; provided by Dr Tingbo Ding, School of Pharmacy, Fudan University) was diluted with $250 \mu$ l DMEM (FBS- and antibiotic-free medium) or $12 \mu 1$ LipoFiter with $238 \mu$ l DMEM. The cells transfected with the SMS2 plasmid were termed the SMS2 group. The cells transfected with the negative plasmid were termed the control group.

After $5 \mathrm{~min}$, the dilutions were mixed together and incubated at $37^{\circ} \mathrm{C}$ for $20 \mathrm{~min}$, then dispensed into each well. After $6 \mathrm{~h}$, the medium was replaced with DMEM containing $10 \%$ FBS and the aforementioned antibiotics. These cells were cultured for $48 \mathrm{~h}$. The control and SMS2 groups were divided separately into two groups, resulting in control, control + DDP, SMS2 and SMS2 + DDP groups. The control + DDP and SMS2 + DDP groups were treated with $3.5 \mathrm{mg} / 1$ DDP. After $24 \mathrm{~h}$, the cells were harvested for analysis.

Western blot analysis. The proteins were extracted using radioimmunoprecipitation assay buffer (CW2333S; Kangwei Century Biotechnology Co., Ltd., Beijing, China), and the protein concentration was measured using the DR4a bicinchoninic acid assay (CW0014; Kangwei Century Biotechnology Co., Ltd.). Equal quantities of cleared lysates ( $\sim 50 \mu \mathrm{g}$ protein) were separated by SDS-PAGE (10\% gel), and then transferred onto polyvinylidene fluoride membranes (EMD Millipore,

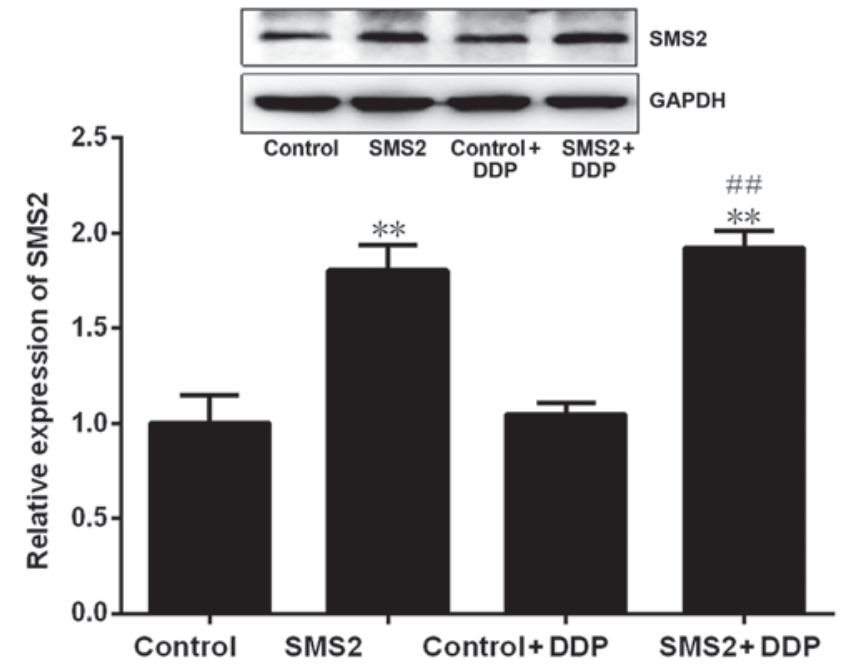

Figure 1. Expression of SMS2 in HepG2 cells. $\mathrm{n}=3 ;{ }^{* *} \mathrm{P}<0.001$ vs. control group; ${ }^{\# /} \mathrm{P}<0.001$ vs. control + DDP group. DDP, cisplatin; SMS2, sphyngomyelin synthase 2 .

Billerica, MA, USA). Equal transfer was validated by staining with Ponceau red for $15 \mathrm{~min}$ at room temperature. The membranes were blocked with $10 \%$ skimmed milk in Tris-buffered saline (TBS), and then incubated with primary antibodies in TBS containing $0.05 \%$ Tween-20, $2 \%$ bovine serum albumin (A8010; Solarbio Bioscience \& Technology Co., Ltd.) and $0.05 \%$ sodium azide overnight at $4^{\circ} \mathrm{C}$. The following antibodies were used at the indicated dilutions: SMS2 (1:1,000; sc-34048; Santa Cruz Biotechnology, Inc., Dallas, TX, USA), caspase-3 (1:1,000; 19677-1-AP; ProteinTech Group, Inc., Chicago, IL, USA), avian myelocytomatosis viral oncogene homolog (c-Myc) (1:1,000; AF0358; Affinity Biosciences, Jiangsu, China), DR4 (1:1,000; AF0304; Affinity Biosciences) and DR5 (1:1,000; DF6368, Affinity Biosciences) GAPDH (1:10,000; 60004-1-Ig; ProteinTech Group, Inc.). Secondary horseradish peroxidase-coupled antibodies [mouse (SA00001-1) and rabbit (SA00001-2); ProteinTech Group, Inc.] were used at 1:10,000 in 10\% skimmed milk in TBS containing $0.05 \%$ Tween-20. Signals were detected using an enhanced chemiluminescence reagent (CW0049M; Kangwei Century Biotechnology Co., Ltd.) and an autoradiography system (Chemiluminescence Imaging system; CLINX Science Instruments Co., Ltd., Shanghai, China) (14). Each assay was repeated at least three times.

Cell viability assays. For cell viability assays, the cells (control and SMS2 groups; $1.5 \times 10^{4}$ cells/well) were plated into flat-bottomed 96-well plates. Next, $3.5 \mathrm{mg} / 1 \mathrm{DDP}$ was added into these cells, and the cells were cultured in $37^{\circ} \mathrm{C}$ to measure viability. After incubation for $24 \mathrm{~h}, 20 \mu \mathrm{l}$ Cell Counting kit-8 (CCK-8) solution (Beijing Zoman Biotechnology Co., Ltd., Beijing, China) was added into each well at $37^{\circ} \mathrm{C}$ in the dark for $2 \mathrm{~h}$. The absorbance of each well was measured using a microplate reader at an absorbance of $450 \mathrm{~nm}$. Each assay was repeated at least three times.

Flow cytometric analysis for apoptosis. The proportion of apoptotic cells was determined by flow cytometry, as described previously (15). In brief, cells from the control and 


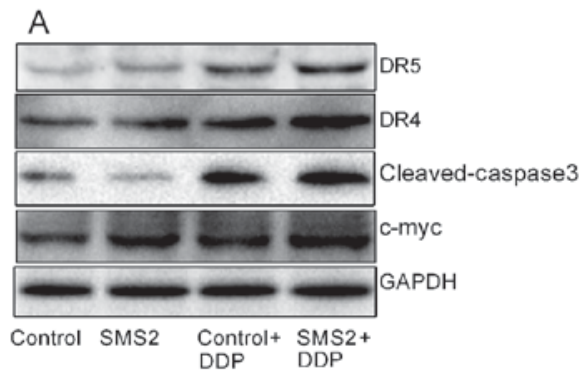

B

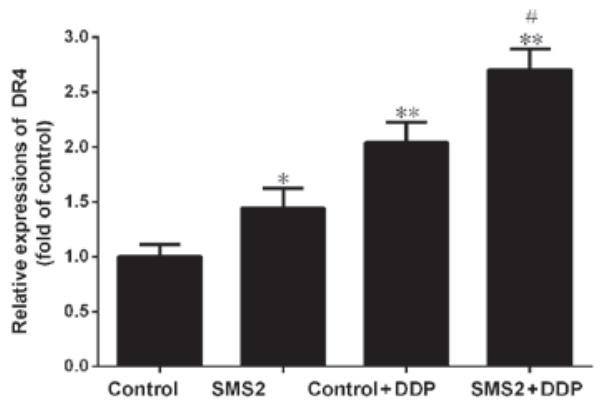

D

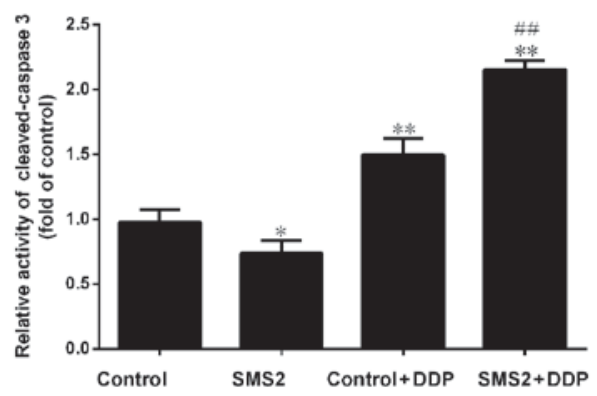

C

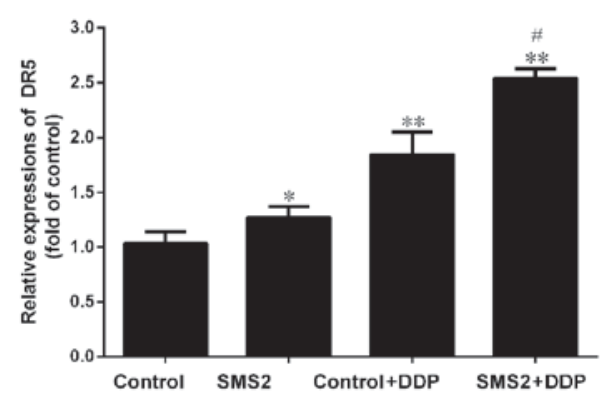

E

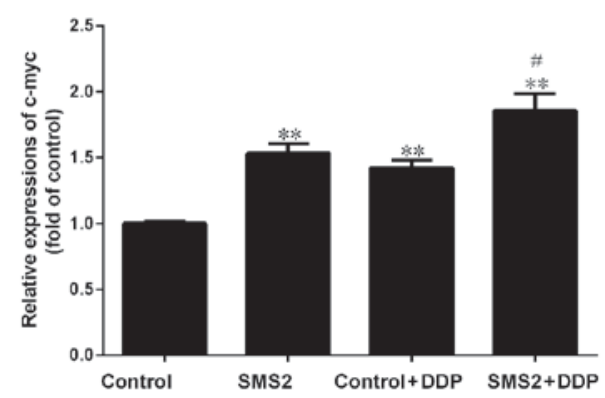

Figure 2. Expression of DR4, DR5, cleaved caspase-3 and c-Myc in HepG2 cells. (A) The protein expression of DR4, DR5, cleaved caspase-3 and c-Myc was investigated using western blotting. Quantification and statistical analysis was performed on (B) DR4, (C) DR5, (D) cleaved caspase-3 and (E) c-Myc. $\mathrm{n}=3$, ${ }^{*} \mathrm{P}<0.05,{ }^{* *} \mathrm{P}<0.001$ vs. control group; ${ }^{\#} \mathrm{P}<0.05,{ }^{\# \#} \mathrm{P}<0.001$ vs. control + DDP group. DR. death receptor; DDP, cisplatin; SMS2, sphyngomyelin synthase 2 ; c-Myc, avian myelocytomatosis viral oncogene homolog.

SMS2 groups $\left(1 \times 10^{6}\right.$ cells $\left./ \mathrm{ml}\right)$ were seeded in 6-well plates and cultured for $12 \mathrm{~h}$, treated with DDP at $3.5 \mathrm{mg} / \mathrm{l}$ for $24 \mathrm{~h}$ and collected. The cells were then washed twice and subsequently analyzed apoptosis by flow cytometry (BD Biosciences, Franklin Lakes, NJ, USA) according to the protocol of the apoptosis assay kit (Promega Corporation, Madison, WI, USA). FlowJo Software (version 7.6; FlowJo LLC, Ashland, OR, USA) was used to analyze these data. Each assay was repeated in triplicate.

Statistical analysis. All data are reported as the mean \pm standard deviation. Student's t-test was used for single comparisons. For multiple comparisons, one-way analysis of variance with Tukey's or Games-Howell post hoc analysis was used. $\mathrm{P}<0.05$ was considered to indicate a statistically significant difference.

\section{Results}

Identification of SMS2 overexpression cell model. To construct a cell model where SMS2 is overexpressed, HepG2 cells were transfected with the SMS2 plasmid. Western blotting revealed that SMS2 expression was significantly upregulated in the SMS2 and SMS2 + DDP groups compared with control and control + DDP group, respectively $(\mathrm{P}<0.001, \mathrm{n}=3$; Fig. 1$)$. The expression of SMS2 in the SMS2 and SMS2 + DDP groups was increased 0.81- and 0.92-fold compared with the control or control + DDP group, respectively. These results demonstrated that transfection with the SMS2 plasmid was able to effectively augment SMS2 protein expression.

Expression of DR4 and DR5. DR4 and DR5 are involved in DDP-induced apoptosis. Therefore, the present study analyzed the expression of DR4 and DR5 by western blot analysis (Fig. 2). The results indicated that upregulation of SMS2 was able to modulate the expression of DR4 and DR5 compared with the control group, respectively. The expression of DR4 and DR5 was increased $\sim 0.48$ and 0.27 -fold in the SMS2 group compared with the control group, respectively $(\mathrm{P}<0.05, \mathrm{n}=3$; Fig. 2B and $\mathrm{C})$. However, when the control and SMS2 groups were treated with DDP, the expression of DR4 and DR5 was also induced. The expression of DR4 in the control + DDP group was significantly increased 1.02-fold 
A

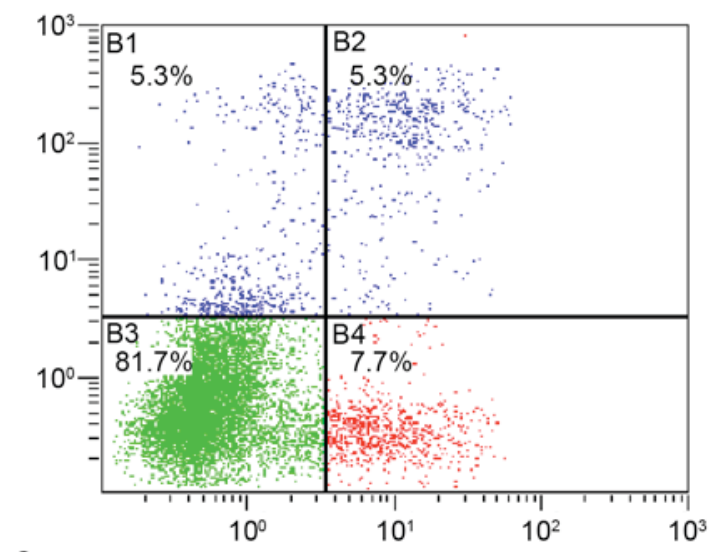

C

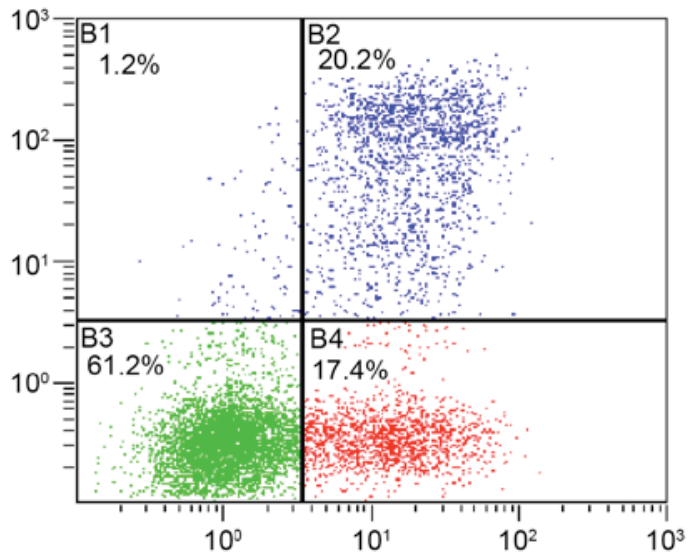

$E$

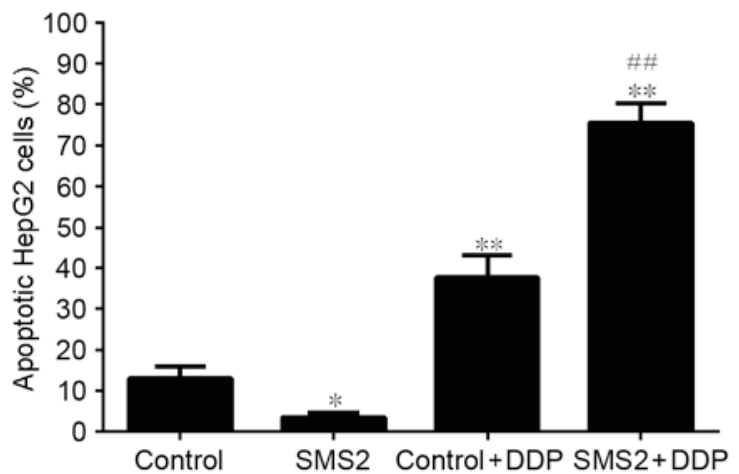

B
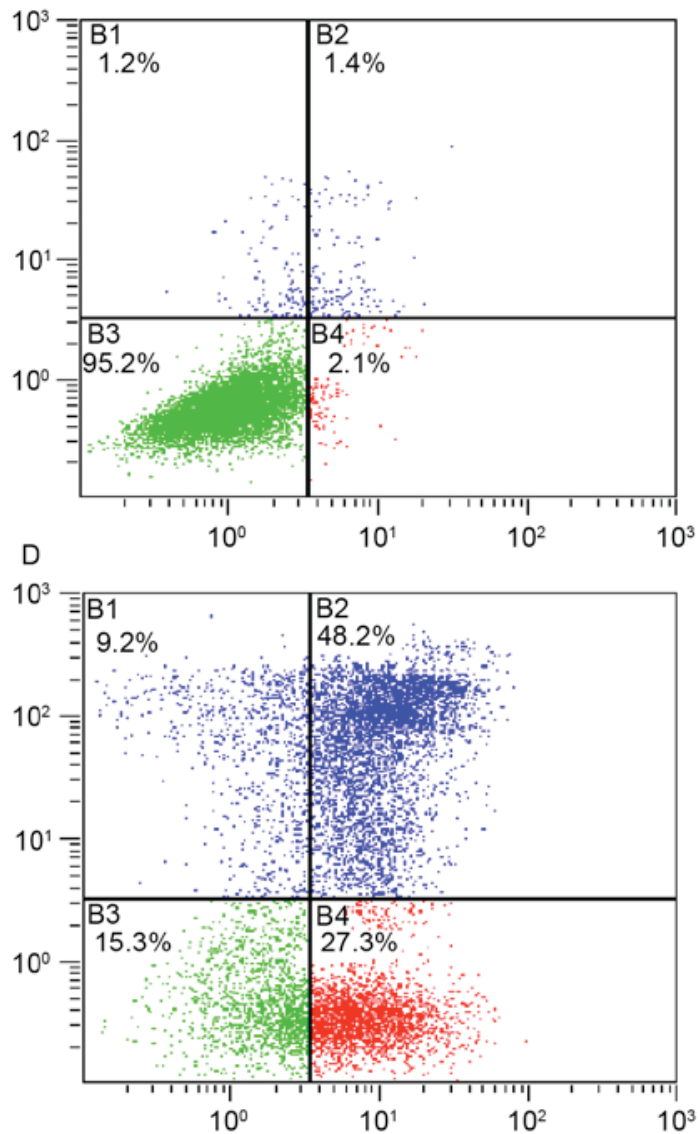

$\mathrm{F}$

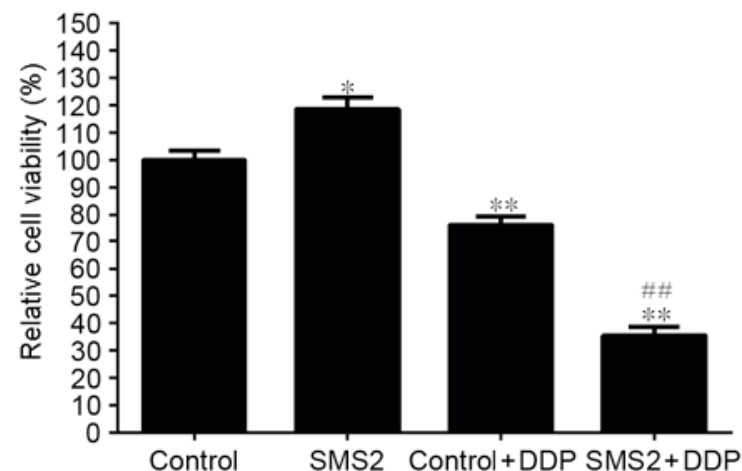

Figure 3. Apoptosis and viability of HepG2 cells. The apoptosis of (A) control, (B) SMS2, (C) control + DDP and (D) SMS2 + DDP groups. (E) Quantification of apoptosis and $(\mathrm{F})$ viability of HepG2 cells. $n=3$, apoptosis analysis; $n=9$, viability assays; ${ }^{\prime} \mathrm{P}<0.05 ;{ }^{* *} \mathrm{P}<0.001$ vs. control group; ${ }^{\# *} \mathrm{P}<0.05$ vs. control $+\mathrm{DDP}$ group. DDP, cisplatin; SMS2, sphyngomyelin synthase 2.

compared with the control group $(\mathrm{P}<0.001, \mathrm{n}=3$; Fig. 2B). By contrast, the expression of DR5 in the control + DDP group only increased $\sim 0.81$-fold compared with the control group (Fig. 2C). Notably, the expression of DR 4 and DR 5 in SMS2 + DDP group compared with the control group was increased 1.64 and 1.47 -fold, respectively $(\mathrm{P}<0.001, \mathrm{n}=3)$. Additionally, the expression of DR4 and DR5 in the SMS2 + DDP group was upregulated compared with the control group $(\mathrm{P}<0.05, \mathrm{n}=3)$.

Effect of SMS2 expression on apoptosis. To analyze the effect of SMS2 expression on the DDP-induced apoptosis of HepG2 cells, the viability of cells was analyzed (Fig. 3). As shown in Fig. 3F, the expression of SMS2 was able to increase the viability of HepG2 cells by $\sim 18.3 \%$ compared with the control group $(\mathrm{P}<0.05, \mathrm{n}=9)$. However, when the cells were treated with DDP, the viability of the cells in the control + DDP and SMS2 + DDP groups compared with the control group was significantly decreased by $23.7 \%(\mathrm{P}<0.05, \mathrm{n}=9)$ and $64.4 \%$ $(\mathrm{P}<0.001, \mathrm{n}=9)$, respectively. Moreover, compared with the control + DDP group, the viability of the cells in the SMS2 + DDP group significantly decreased, by $\sim 40.7 \%(\mathrm{P}<0.001$, $\mathrm{n}=9$ ). These observations were confirmed by flow cytometric analysis. The percentage of apoptotic cells in the control, SMS2, control + DDP and SMS2 + DDP groups were 13.0, 
3.5, 37.6 and $75.5 \%$, respectively (Fig. 3A-D). The proportion of apoptotic cells in the SMS2 + DDP group increased by $\sim 37.9 \%$ compared with the control + DDP group (Fig. 3E, $\mathrm{P}<0.001, \mathrm{n}=3$ ). The levels of cleaved caspase-3 and $\mathrm{c}-\mathrm{myc}$ were assessed using western blotting (Fig. 2A). The levels of cleaved caspase- 3 in the SMS2 + DDP group was increased by $115.3 \%$ compared with the control + DDP group $(\mathrm{P}<0.001$, $\mathrm{n}=3$; Fig. 2D). The levels in the control + DDP group were significantly increased compared with the control group $(\mathrm{P}<0.001, \mathrm{n}=3$; Fig. 2D), where expression was increased by $37.2 \%(\mathrm{P}<0.001, \mathrm{n}=3$; Fig. 2D). Expression of SMS2 was able to increase the sensitivity of HepG2 cells to DDP treatment.

Expression of $c-M y c$. Furthermore, the present study continued to investigate the expression of c-Myc. As presented in Fig. 2E, the expression of c-Myc in the SMS2 group was increased 0.48-fold, compared with the control group $(\mathrm{P}<0.01, \mathrm{n}=3)$. However, when the HepG2 cells were treated with DDP, the expression of c-Myc in the control + DDP and SMS2 + DDP groups was increased compared with the control group $(\mathrm{P}<0.001, \mathrm{n}=3)$. Notably, the degree of increase in c-Myc expression in the SMS2 + DDP group compared with the control was greater compared with the increase in the control + DDP group compared with the control $(\mathrm{P}<0.05, \mathrm{n}=3)$.

\section{Discussion}

The results of the present study indicated that the overexpression of SMS2 was able to slightly increase the viability of HepG2 cells and increase the expression of DR4, DR5 and c-Myc. Overexpression of SMS2 was also able to increase the sensitivity of the HepG2 cells to DDP.

SMS2 is a key enzyme involved in the biosynthesis of sphingomyelin and is also involved in the production of diacylglycerol (DAG), which is a second messenger that can induce cell proliferation (16). Overexpression of SMS2 may therefore increase the cellular levels of DAG and induce growth signal transmission.

Moreover, the proliferation of HepG2 cells may also be associated with c-Myc. c-Myc is a proto-oncogene which is a key regulator of cell proliferation, apoptosis and cellular differentiation (17). However, in the present study, the expression of SMS2 was able to upregulate the expression of c-Myc, which would lead to the viability of HepG2 cells. A number of studies have reported that upregulation of SMS is able to induce cell proliferation $(18,19)$. For example, chronic myelogenous leukemia K562 cells have high SMS activity, and when SMS1 expression was downregulated using small interfering RNAs, cell apoptosis was induced (18). This result indicates that maintaining the SMS activity was necessary to proliferation of K562 cells (18).

c-Myc not only modulates the expression of growth genes, but also participates in the expression of DR4 and DR5, which are associated with apoptosis $(20,21)$. When c-Myc was overexpressed, the expression of DR4 and DR5 were also increased, which led to an increase in the sensitivity to tumor necrosis factor-related apoptosis-inducing ligand (TRAIL), as DR4 and DR5 are TRAIL receptors $(20,21)$. This may overcome multidrug resistance in human ovarian, breast and gastric carcinoma cells $(20,21)$.
Similar to TRAIL, DDP is also able to induce apoptosis through the DR4 and DR5 signaling pathways (6,7). DDP is able to trigger apoptosis through the DR4 and DR5 signal pathways in H460 cells, independent of TRAIL and the mitochondrial apoptotic pathway (9). Therefore, in the present study, it was hypothesized that overexpression of SMS2 was able to alter the expression of DR4 and DR5, and increased the expression of c-Myc, as well as increasing the sensitivity of the cells to DDP. However, DDP is also able to increase the expression of DR4 and DR5 to induce cell apoptosis $(7,21)$. In the present study, it was demonstrated that treatment with DDP was able to significantly increase the expression of DR4 and DR5 in HepG 2 cells, and the increase in the expression of DR4 and DR5 was higher in the SMS2 + DDP group compared with the control + DDP group. Other studies reported that overexpression of SMS2 is able to increase the sensitivity of cancer cells to antitumor drugs. For example, Ding et al (22) found that when SMS1 and SMS2 were overexpressed in THP-1 cells, apoptosis was increased via tumor necrosis factor or lipopolysaccharide. 2-hydroxyoleic acid (2OHOA) is a potent antitumor compound. It has been demonstrated that 2OHOA is able to regulate SMS activity in tumor cells, which is a critical upstream event (23).

Moreover, the increase in apoptosis induced by DDP may be associated with the lipid raft, which act as microdomains of the plasma membrane and are enriched in cholesterol and sphingolipids. Lipid rafts have an important function in signaling, vesicular transporting, interaction with pathogens and viral infection (24). SM is a type of sphingolipid that can affect lipid raft structure and function. DR4 and DR5 localize to lipid rafts and can accelerate the internalization of TRAIL $(2,6,8)$. Certain studies hypothesized that the localization of DR4 and DR5 to the lipid raft was responsible for the transduction of intrinsic and extrinsic apoptosis signaling pathways in response to DDP treatment $(8,9)$. Furthermore, in the present study, SMS2 was overexpressed in HepG2 cells, which may alter the structure of the lipid raft, and the relocalization of DR4 and DR5 to the lipid raft. Therefore, overexpression of SMS2 not only increased apoptosis of HepG2 cells by increasing the expression of DR4 and DR5, but may also have caused the relocalization of DR4 and DR5 to the lipid raft.

To conclude, the present study found that overexpression of SMS2 increased the sensitivity of HepG2 cells to DDP through c-Myc and an increase in the expression of DR4 and DR5. This increased sensitivity, if reflected in patients, would decrease intrinsic and acquired resistance, and severe side effects of $\mathrm{HB}$ chemotherapy in pediatric malignancies.

\section{Acknowledgements}

The present research was supported by grants from the National Natural Science Foundation of China (grant no. 81560151) and Jiangxi Provincial Department of Science and Technology (grant no. 20142BAB205014).

\section{References}

1. Yuan XJ, Wang HM, Jiang H, Tang MJ, Li ZL, Zou X, Fang YJ, Pan C, Tou JF, Zhang KR, et al: Multidisciplinary effort in treating children with hepatoblastoma in China. Cancer Lett 375: $39-46,2016$. 
2. van As JW, van den Berg H and van Dalen EC: Medical interventions for the prevention of platinum-induced hearing loss in children with cancer. Cochrane Database Syst Rev 9: CD009219. 2016.

3. Zhu S, Pabla N, Tang C, He L and Dong Z: DNA damage response in cisplatin-induced nephrotoxicity. Arch Toxicol 89: 2197-2205, 2015

4. Fujikawa Y, Kawanishi M, Kuraoka I and Yagi T: Frequencies of mutagenic translesion DNA synthesis over cisplatin-guanine intra-strand crosslinks in lacZ plasmids propagated in human cells. Mutat Res Genet Toxicol Environ Mutagen 770: 23-28, 2014.

5. Chipuk JE, Kuwana T, Bouchier-Hayes L, Droin NM, Newmeyer DD, Schuler M and Green DR: Direct activation of Bax by $\mathrm{p} 53$ mediates mitochondrial membrane permeabilization and apoptosis. Science 303: 1010-1014, 2004.

6. Vondálová Blanárová O, Jelínková I, Szöor A, Skender B, Soucek K, Horváth V, Vaculová A, Andera L, Sova P, Szöllosi J, et al: Cisplatin and a potent platinum(IV) complexmediated enhancement of TRAIL-induced cancer cells killing is associated with modulation of upstream events in the extrinsic apoptotic pathway. Carcinogenesis 32: 42-51, 2011.

7. Zhu X, Zhang K, Wang Q, Chen S, Gou Y, Cui Y and Li Q Cisplatin-mediated c-Myc overexpression and cytochrome c (cyt c) release result in the up-regulation of the death receptors DR4 and DR5 and the activation of caspase-3 and caspase 9 , likely responsible for the TRAIL-sensitizing effect of cisplatin. Med Oncol 32: 133, 2015.

8. Song JH, Tse MC, Bellail A, Phuphanich S, Khuri F, Kneteman NM and Hao C: Lipid rafts and non-rafts mediate tumor necrosis factor related apoptosis-inducing ligand induced apoptotic and nonapoptotic signals in non small cell lung carcinoma cells. Cancer Res 67: 6946-6955, 2007.

9. Paul I, Chacko AD, Stasik I, Busacca S, Crawford N, McCoy F, McTavish N, Wilson B, Barr M, O'Byrne KJ, et al: Acquired differential regulation of caspase- 8 in cisplatin-resistant non-small-cell lung cancer. Cell Death Dis 3: e449, 2012.

10. Yeang C, Ding T, Chirico WJ and Jiang XC: Subcellular targeting domains of sphingomyelin synthase 1 and 2. Nutr Metab (Lond) 8: 89, 2011.

11. Taniguchi $\mathrm{M}$ and Okazaki $\mathrm{T}$ : The role of sphingomyelin and sphingomyelin synthases in cell death, proliferation and migration-from cell and animal models to human disorders. Biochim Biophys Acta 1841: 692-703 2014.

12. Slotte JP: Biological functions of sphingomyelins. Prog Lipid Res 52: 424-437, 2013.

13. López-Terrada D, Cheung SW, Finegold MJ and Knowles BB: Hep G2 is a hepatoblastoma-derived cell line. Hum Pathol 40: $1512-1515,2009$.
14. Yan N, Ding T, Dong J, Li Y and Wu M: Sphingomyelin synthase overexpression increases cholesterol accumulation and decreases cholesterol secretion in liver cells. Lipids Health Dis 10: 46, 2011.

15. Huang CR, Jin ZX, Dong L, Tong XP, Yue S, Kawanami T, Sawaki T, Sakai T, Miki M, Iwao H, et al: cisplatin augments FAS-mediated apoptosis through lipid rafts. Anticancer Res 30: 2065-2071, 2010.

16. Luberto $C$ and Hannun YA: Sphingomyelin synthase, a potential regulator of intracellular levels of ceramide and diacylglycerol during SV40 transformation. Does sphingomyelin synthase account for the putative phosphatidylcholine-phospholipase C. J Biol Chem 273: 14550-14559, 1998.

17. Sumi T, Tsuneyoshi N, Nakatsuji N and Suemori H: Apoptosis and differentiation of human embryonic stem cells induced by sustained activation of c-Myc. Oncogene 26: 5564-5576, 2007.

18. Burns TA, Subathra M, Signorelli P, Choi Y, Yang X, Wang Y, Villani M, Bhalla K, Zhou D and Luberto C: Sphingomyelin synthase 1 activity is regulated by the BCR-ABL oncogene. J Lipid Res 54: 794-805, 2013.

19. Wesley UV, Hatcher JF and Dempsey RJ: Sphingomyelin synthase 1 regulates Neuro-2a cell proliferation and cell cycle progression through modulation of p27 expression and Akt signaling. Mol Neurobiol 51: 1530-1541, 2015.

20. Kim DY, Kim MJ, Kim HB, Lee JW, Bae JH, Kim DW, Kang CD and Kim SH: Suppression of multidrug resistance by treatment with TRAIL in human ovarian and breast cancer cells with high level of c-Myc. Biochim Biophys Acta 1812: 796-805, 2011.

21. Kondo K, Yamasaki S, Sugie T, Teratani N, Kan T, Imamura M and Shimada Y: Cisplatin-dependent upregulation of death receptors 4 and 5 augments induction of apoptosis by TNF-related apoptosis-inducing ligand against esophageal squamous cell carcinoma. Int J Cancer 118: 230-242, 2006.

22. Ding T, Li Z, Hailemariam T, Mukherjee S, Maxfield FR, Wu MP and Jiang XC: SMS overexpression and knockdown: Impact on cellular sphingomyelin and diacylglycerol metabolism, and cell apoptosis. J Lipid Res 49: 376-385, 2008.

23. Barceló-Coblijn G, Martin ML, de Almeida RF, Noguera-Salvà MA, Marcilla-Etxenike A, Guardiola-Serrano F, Lüth A, Kleuser B, Halver JE and Escribá PV: Sphingomyelin and sphingomyelin synthase (SMS) in the malignant transformation of glioma cells and in 2-hydroxyoleic acid therapy. Proc Natl Acad Sci 108: 19569-19574, 2011.

24. Simons K and Ikonen E: Functional rafts in cell membranes. Nature 387: 569-572, 1997. 